\title{
Electrocardiograph and Chest X-ray in Prediction of Left Ventricular Systolic Dysfunction
}

\author{
Basnet BK, ${ }^{1}$ Manandhar K,' Shrestha R, ${ }^{2}$ Shrestha $\mathrm{S}^{3}$, Thapa $\mathrm{M}^{4}$ \\ 'Department of Medicine, Bir Hospital, NAMS, ${ }^{2}$ Kasthamandap Health Care Centre, Kathmandu, ${ }^{3}$ Kakani Primary Health \\ Centrez, Nuwakot, ${ }^{4}$ T.U. Teaching Hospital, Maharigunj, Kathmandu.
}

\section{ABSTRACT}

Introduction: Echocardiography is the definitive diagnostic tool for left ventricular systolic dysfunction. But it's expensive and requires trained manpower and thus might not be available in the primary care set up. ECG and Chest $X$ ray, the more basic investigations, may help diagnose LVSD or at least streamline those who absolutely require echocardiography in primary care setup.

Methods: ECG, Chest X ray and Echocardiography along with clinical assessment were performed on 35 patients with some form of complaints related to heart. The inferences on systolic function obtained from ECG, Chest $\mathrm{X}$ ray were compared with Echocardiography findings.

Results: Out of 35 participants, 25 had left ventricular ejection fraction less than $45 \%, 28$ had abnormal ECG, 30 had cardiomegaly in chest X-ray. A set of pre-selected ECG abnormalities had a sensitivity of $100 \%$ (83.4-100), specificity of 70\% (35.4-91.9) and a positive predictive value of $89.3 \%$ (70.6-97.2) in diagnosing LVSD. Likewise, the figures were $92 \%$ (72.5-98.6), 30\% (8.1-64.6) and 76.7\% (57.3-89.4) respectively for a cardio-thoracic ratio of more than 0.5 in chest $\mathrm{X}$-ray.

Conclusions: Although, ECG and Chest X ray could not replace Echocardiography, they could very well give an idea of the systolic function of an individual and suggest the need or no need for an echo-study in primary care setup.

Key Words: chest X-ray, echocardiography, electrocardiography, left ventricular systolic dysfunction

Correspondence:
Dr. Bhupendra Kumar Basnet
Department of Medicine
Bir Hospital, NAMS, Kathmandu.
Email: bkb_dr@yahoo.com
Phone: $98 \overline{5} 1059400$




\section{INTRODUCTION}

Left ventricular systolic dysfunction (LVSD) is said to occur when there is difficulty in emptying of left ventricle and it marks the beginning of chronic heart failure.

Quantitatively, left ventricular ejection fraction (EF) becomes less than $45 \%$ in adults. ${ }^{1,2}$

Many patients may remain asymptomatic even with significant compromise in their systolic function because of compensatory mechanisms involving the autonomic nervous system, neuro-hormones and changes in cardiac structure and function. Only echocardiography provides definitive assessment of systolic function. But, it requires more resources and expert man power which is not available at all levels of primary health care. LVSD is the most common precursor of CHF. Early detection \& treatment with ACE inhibitor could delay progression, imporve quality of life $\&$ increased survival. Similarly reduced hospitalization \& heavy health expenses.

In such scenario, it would be a great help if there were any common and routine investigation which could reflect systolic dysfunction. Electrocardiography (ECG) and chest $X$-ray are simple and cheap investigations easily available at all primary health care settings and they might serve the purpose. The aim of this study was to compare ECG and Chest $X$-ray findings with Echocardiography as a standard to detect the systolic dysfunction.

\section{METHODS}

This is a cross sectional observational study conducted in two tertiary level hospitals viz Bir hospital and Tribhuvan university teaching hospital, Kathmandu in the year 2003. Ethical approval and patient consent were taken.

Thirty five patients, who met the inclusion criteria and who consented for the study, were selected at convenience. Patients aged more than 40 and presenting with signs and symptoms of congestive cardiac failure or any heart disease were considered compatible for the study. But those with advanced cardiac failure on treatment, with signs and symptoms of congenital or rheumatic heart disease or respiratory diseases like pneumothorax, pneumonia or cor-pulmonale, and those unfit for transfer to echocardiography were excluded from the study.
Clinical history, complete physical examination, electrocardiography, chest radiography, and echocardiography were done for all the cases. Clinical history focused on past or present palpitation, chest pain, dyspnoea, fatigue, weakness, treatment of heart disease, and referral to intensive care unit. Likewise, complete physical examination of patients was done with special emphasis on the relationship between resting supine heart rate and simultaneous diastolic blood pressure measured after 10 minutes rest. ${ }^{2}$ Patients presenting with symptoms of breathlessness were classified according to New York Heart Association Classification of heart failure symptom severity.

A supine 12-lead ECG was recorded at admission. Abnormal ECG was taken in favor of LVSD including Left bundle branch block (LBBB), pathological $\mathrm{Q}$ wave, atrial fibrillation (AF), ST-T changes, Left ventricular hypertrophy (LVH) and Left axis deviation (LAD). ${ }^{2-4}$ Likewise, Posteroanterior chest radiography was taken in full inspiration at admission and evaluated for the size of heart and features of pulmonary venous congestion or edema. Heart size was assessed as ratio between the maximum transverse diameter of heart and the width of the thorax. A cardiothoracic ratio of more than 0.5 was considered to indicate cardiomegaly. ${ }^{5}$ Echocardiography was regarded as standard test to diagnose LVSD evaluated by left ventricular ejection fraction (LVEF) equal to or less than $45 \%$ in adult. ${ }^{1,2,6,7}$ Left ventricular ejection fraction was measured by modified Simpson's method.

Each of the observations - history and examination, ECG, chest X-ray and echocardiography - was performed by a different individual, echocardiography in particular by an expert, blinded to the findings of other investigations. Statistical analysis was done with Statistical Package for Social Sciences (SPSS) version 10 for windows.

\section{RESULTS}

There were thirty-five patients with 20 males and 15 females. The mean age of the participants was 62.66 \pm 10.7 years, majority in the age group 60 to 69 . Seventeen $(48.6 \%)$ of them were hypertensive, 12 $(34.3 \%)$ were diabetic, 24 (68.6\%) had ischemic heart disease and 26 (74.3\%) were smokers.

\section{JNMA | Val 48}


Table 1. Age and sex distribution of the participants

\begin{tabular}{llll}
\hline Age group (years) & Male & Female & Total (\%) \\
\hline $40-49$ & 3 & 2 & $5(14.3)$ \\
$50-59$ & 5 & 2 & $7(20)$ \\
$60-69$ & 9 & 7 & $16(45.7)$ \\
$70-79$ & 2 & 2 & $4(11.4)$ \\
$>80$ & 1 & 2 & $3(8.6)$ \\
TOTAL & 20 & 15 & $35(100)$ \\
\hline
\end{tabular}

Breathlessness $(85.71 \%)$ was the most common presenting complaint. Among them, majority belonged to NYHA class IV $(15,50 \%)$, NYHA class III $(7,23.3 \%)$ and NYHA II $(8,26.6 \%)$. Likewise, the most common physical sign was resting supine heart rate more than simultaneous diastolic blood pressure (HR $>$ DBP) $(74.3 \%)$.

Table 2. Clinical features of patients

\begin{tabular}{lcll}
\hline Clinical Symptoms & Number (\%) & Clinical signs & Number (\%) \\
\hline Breathlessness & $30(85.7)$ & HR $>$ DBP & $26(74.3)$ \\
Chest discomfort/heaviness $29(82.9)$ & Basal crepitations & $25(71.4)$ \\
Weakness/fatigue & $25(71.4)$ & JVP' & $20(57.1)$ \\
Orthopnoea & $18(51.5)$ & Edema & $19(54.3)$ \\
Palpitation & $13(37.1)$ & Displaced apex beat & $17(48.6)$ \\
Leg swelling & $11(31.4)$ & S3† & $14(40)$ \\
Chest Pain (anginal type) & $11(31.4)$ & Palpable liver & $13(37.1)$ \\
PND † & $8(22.9)$ & Pallor & $8(22.8)$ \\
& & Cyanosis & $1(2.9)$ \\
\hline
\end{tabular}

Jugular venous pressure, tthird heart sound, $\uparrow \uparrow$ Paroxysmal nocturnal dyspnoea

On chest radiography, 30 patients had enlarged cardiac shadow, 10 patients had congestion and upper lobe diversion and eight of them had features suggestive of infection. Likewise, 28 (80\%) patients had abnormal 12lead ECG and $25(71.4 \%)$ had left ventricular ejection fraction less than $45 \%$, suggestive of LVSD.
Table 3. Chest $\mathbf{X}$-ray findings at presentation

\begin{tabular}{ll}
\hline X-ray finding & Number (\%) \\
\hline Enlarged cardiac size & $30(85.7)$ \\
Upper lobe diversion & $10(28.6)$ \\
Congestion & $10(28.6)$ \\
Infection & $8(22.9)$ \\
\hline
\end{tabular}

Table 4. Major ECG abnormalities

\begin{tabular}{ll}
\hline ECG abnormality & Number $(\%)$ \\
\hline AF & $2(5.71)$ \\
Q wave (anterior lead) & $8(22.85)$ \\
Q wave (Inferior lead) & $3(8.57)$ \\
LVH & $7(20)$ \\
LBBB & $6(17.14)$ \\
LAD & $8(22.85)$ \\
ST-T changes & $17(48.57)$ \\
\hline
\end{tabular}

study, Gillespie ND et al $^{9}$ found a sensitivity of $71 \%$ (55\%-83\%) and specificity of 92\% (74\%-99\%) which is very different from our findings. However, cardiomegaly in chest $\mathrm{x}$-ray significantly decreases the possibility of having normal left ventricular ejection fraction as is also concluded by other study..$^{10}$ In addition; a normal size heart associated with clinical evidence of chronic heart failure indicates that diagnosis should be reviewed. ${ }^{8}$ Likewise, a set of selected ECG abnormalities had 100\% sensitivity and a specificity of $70 \%$ in diagnosing LVSD. These findings are similar to those of Davie et al. ${ }^{4}$ who reported ECG sensitivity of $94 \%$, specificity of $61 \%$ and positive predictive value of $35 \%$ and negative predictive value of $98 \%$ in diagnosing LVSD. Likewise, another study also provided similar findings ${ }^{9}$ sensitivity $98 \%$, specificity $69 \%$, positive predictive value $85 \%$ and negative predictive value $95 \%$.

Table 5. Comparison of ECG, Chest X-ray with Echocardiography in diagnosis of LVSD

\begin{tabular}{llllllll}
\hline Parameter & \multicolumn{2}{c}{ LVSD } & Total & Sensitivity & \multicolumn{2}{l}{ Specificity } & \multicolumn{2}{c}{ Predictive accuracy } \\
& Yes & No & & (95\% Cl) & (95\% Cl) & Positive & Negative \\
\hline Abnormal ECG & 25 & 3 & 28 & $100(83.4-100)$ & $70(35.4-91.9)$ & $89.3(70.6-97.2)$ & $100(56.1-100)$ \\
Cardiomegaly in X-ray & 23 & 7 & 30 & $92(72.5-98.6)$ & $30(8.1-64.6)$ & $76.7(57.3-89.4)$ & $60 \quad(17-92.7)$ \\
\hline
\end{tabular}

\section{DISCUSSION}

The study showed that ECG and Chest $X$ ray could be used as initial investigations to predict the systolic function, however could not replace echocardiography.

This study showed cardiomegaly with upper lobe diversion in chest radiograph is a very sensitive sign to left ventricular dysfunction, but it's not much specific. One study also showed a poor relation between heart size on chest radiography and left ventricular function. ${ }^{8}$ In another
Among the ECG abnormalities, Q-wave, LBBB, ST-T changes and LVH all were found significant for the diagnosis of LVSD. This is also supported by the findings of Nielsen OW et $\mathrm{al}^{2}$ where he found ECG with Q- wave, LBBB, ST-T changes were significantly $(P<0.012)$ associated with LVSD. All patients with LBBB were found to have LVSD. Das et $\mathrm{al}^{11}$ also reported similar finding. He also added that prolongation of QRS duration more than $170 \mathrm{~ms}$ in presence of LBBB was a marker of significant left ventricular systolic dysfunction. Talreja et al, ${ }^{7}$ also emphasized the presence of LBBB as a strong indicator of LVSD. 
None of the patients with normal ECG had left ventricular systolic dysfunction in this study, similarly a normal ECG was uncommon in patients with heart failure. In a study by Nielsen et al $^{2}$ only one of 60 patients with a normal electrocardiogram had systolic dysfunction. Similarly, Davie et al, ${ }^{4}$ had shown that left ventricular systolic dysfunction was unlikely to be present if ECG was normal or showed any minor abnormalities. When the ECG is normal, it is extremely unlikely to have left ventricular systolic dysfunction or it virtually excludes the chronic heart failure due to left ventricular systolic dysfunction. ${ }^{4}$ Talreja et $\mathrm{al}^{7}$ reported negative correlation of the normal ECG and left ventricular systolic dysfunction and found only two patients with left ventricular systolic dysfunction with normal electrocardiogram. So, ECG seems to be the most useful test in primary care, which is simple and easily available and does not need an expert. This has been found to be true for patients referred for open access echocardiography in a general population, in referred patient with chest pain, dyspnoea and myocardial infarction. ${ }^{2}$

Furthermore, the combination of ECG and Chest X-ray could have a higher predictive value for LVSD. Thus, we believe that the set of these two investigations along with clinical features suggestive of cardiac failure could be valuable to assess left ventricular function in primary care settings, which could not afford echocardiography due to low resources and lack of skilled man power. Primary care centers are the first contact center and serve the majority of the patients. But unfortunately, the primary care centers, especially in developing countries like Nepal, have very limited resources and are not well set up. They provide only very basic diagnostic services and echocardiography is too advanced and expensive for a primary center to afford and utilize. Besides, proper use of echo machine requires a trained person, most appropriately a cardiologist. In a country where, skilled man power is so scarce, having a cardiologist in a primary health center is simply next to impossible. Thus under such circumstances, ECG and chest $X$-ray could serve as a possible alternative in patients clinically suggestive of cardiac failure. They have quite acceptable accuracy in assessing ventricular systolic function and they are cheap and easily available even in the primary health care set-ups. Though they could not be used as a replacement for echocardiography, they could help to streamline those who are likely to have LVSD or to be referred to a higher health center.

\section{CONCLUSIONS}

Abnormal ECG findings, mainly pathological Q-wave, left bundle branch block, ST-T changes and left ventricular hypertrophy and a cardio-thoracic ratio more than 0.5 could reflect LVSD with a high precision among patients with clinical features of heart failure. Thus, ECG and Chest X-ray, though not as an echocardiography replace, could be used to assess ventricular function. They would prove valuable especially in poor resource setting country like Nepal, where echo is not available and affordable in primary health set-ups.

\section{REFERENCES}

1. Francis GS, Gassler JP, Sonnenblick EH. Patho-physiology and diagnosis of heart failure. In: Fuster V, Alexander RW, O'Rourke RA, editors. Hurst's: The Heart. 10th ed. USA: McGraw Hill Companies; 2001. p. 656-7.

2. Nielsen OW, Fischers J, Hilden J, et al. Risk assessment of left ventricular systolic dysfunction in primary care: cross sectional study evaluating a range of diagnostic tests. BMJ. 2000;320:220-3.

3. Schamroth L. An introduction to electrocardiography. 7th edition. Berlin, Germany: Blackwell Science Ltd; 1990.

4. Davie AP, Francis CM, Love MP, et al. Value of the electrocardiogram in identifying heart failure due to left ventricular systolic dysfunction. BMJ. 1996;312:222.

5. Jefferson $\mathrm{K}$ and Rees S. Clinical Cardiac Radiology. $2^{\text {nd }}$ Ed. London: Butterworth-Heinemann Ltd; 1980. p. 6.

6. Thomas JT, Kelly RF, Thomas SJ, et al. Utility of history, physical examination, electrocardiogram and chest radiograph for differentiating normal from decreased systolic function in patients with heart failure. Am J Med. 2002;112(6):496-7.
7. Talreja D, Gruver C, Sklenar J, et al. Efficient utilization of echocardiography for the assessment of left ventricular systolic function. Am Heart J. 2000;139(3):394-8.

8. Cleland JGF. Diagnosis of heart failure. Heart. 1998;79:SP10-S16.

9. Gillespie ND, McNeill G, Pringle T, et al. Cross sectional study of contribution of clinical assessment and simple cardiac investigations to the diagnosis of left ventricular systolic dysfunction in patients admitted with acute dyspnoea. BMJ. 1997;314:936.

10. Rihal CS, Davis KB, Kennedy JW, Gersh BJ. The Utility of clinical, electrocardiographic, and roentgenographic variables in the prediction of left ventricular function. Am J Cardiol. 1995;75(4):220-3.

11. Das MK, Cheriparambil K, Bedi A, et al. Prolonged QRS duration (QRS $3170 \mathrm{~ms}$ ) and left axis deviation in the presence of left bundle branch block: A marker of poor left ventricular systolic? American Heart Journal. 2001;142(5):756-9. 